\title{
Effect of Intramolecular Hydrogen Bonds on the Single- Chain Elasticity of Poly(vinyl alcohol): Evidencing the Synergistic Enhancement Effect at the Single-Molecule Level
}

Yu Bao,* Xiaobo Huang, Jun Xu, and Shuxun Cui*

Key Lab of Advanced Technologies of Materials, Ministry of Education of China, Southwest Jiaotong University, Chengdu 610031, China.

\section{The detailed generation of the QM-FRC fitting curve of PE}

The detailed generation of the QM-FRC fitting curve of PE has been described in the literature. ${ }^{1}$ In brief, for common synthetic polymers with $\mathrm{C}-\mathrm{C}$ backbones at zero force, the bond length and bond angle are fixed. It is expected that the $\mathrm{C}-\mathrm{C}$ bond can rotate freely around the neighboring bond. Therefore, it is anticipated that the real structure of the polymer chain can be described well by the FRC model. The relationship between the extension $(R)$ of a polymer chain with the FRC model and the stretching force $(F)$ can be written in a good approximation as:

$$
R=L[F]\left[1-k_{\mathrm{B}} T /\left(2 F l_{b}\right)\right]
$$

where $L[F]$ is the contour length of the chain at a given stretching force, $l_{b}$ is the length of the rotating unit, $k_{\mathrm{B}}$ is the Boltzmann constant, and $T$ is the temperature on the Kelvin scale.

The theoretical elasticity of a propane molecule has been obtained by ab-initio QM calculations on the HF-TZV-MP2 level. ${ }^{2}$ Because the minor changes of the bond 
angles and lengths were considered in the QM calculations, the result can be easily rewritten to describe the theoretical elasticity of single PE chain. The chain elasticity is nonlinear, which can be expressed in a polynomial expansion as follows:

$$
\begin{gathered}
F=\sum_{n=1}^{3} \gamma_{n}\left(L[F] / L_{0}-1\right)^{n} \\
\gamma_{1}=28.7 \mathrm{nN}, \gamma_{2}=-42.0 \mathrm{nN}, \gamma_{3}=16.9 \mathrm{nN}
\end{gathered}
$$

where $L_{0}$ is the contour length of the polymer chain at zero force, $\gamma_{1}$ is the linear elastic modulus of the repeating unit, and the other two coefficients are nonlinear corrections that become important at the higher force range.

As described in eq 1, the FRC model has two free parameters, i.e., the contour length of the polymer chain $L[F]$, and the length of the rotating unit $l_{b}$. By introducing $L_{0}$ into the models, we can modify the FRC model as follows:

$$
R / L_{0}=\left(L[F] / L_{0}\right) \cdot\left[1-k_{B} T /\left(2 F l_{b}\right)\right]
$$

where $R / L_{0}$ is the normalized extension of a polymer chain.

The modified FRC model, which is integrated with the theoretical single-chain elasticity from QM calculations, is called the QM-FRC model. For a polymer chain being stretched, $L[F] / L_{0}$ should be an ergodic value in the proper range (from 1 to the value corresponding to the rupture of the polymer bridge). Assuming that the toplimit of the external force is $2000 \mathrm{pN},{ }^{3}$ the upper limit for $L[F] / L_{0}$ of $\mathrm{PE}$ is about 1.078 according to eq 2 . In the range from 1 to 1.078 , any value of $L[F] / L_{0}$ is reasonable and corresponds to a mapping value of $F$ in the fitting curve. Since $L[F] / L_{0}$ is an ergodic value in the range of $1-1.078$, the QM-FRC model now has only one free parameter left $\left(l_{b}\right)$ 
The optimum value of $l_{b}$ for polymers with $\mathrm{C}-\mathrm{C}$ backbones has been determined as $0.154 \mathrm{~nm}$ in the literature. ${ }^{1}$ Thus, there is no free parameter left in eq 3 . The QMFRC fitting curve of PE can be generated as described below: When the value of $L[F] / L_{0}$ varies from 1 to 1.078 , any value of $L[F] / L_{0}$ will correspond to a mapping point in the QM-FRC fitting curve. For this point, $F$ can be calculated with eq 2 , and then $R / L_{0}$ can be calculated with eq 3. In this way, the whole QM-FRC fitting curve of PE is generated.

\section{Supporting Figures}

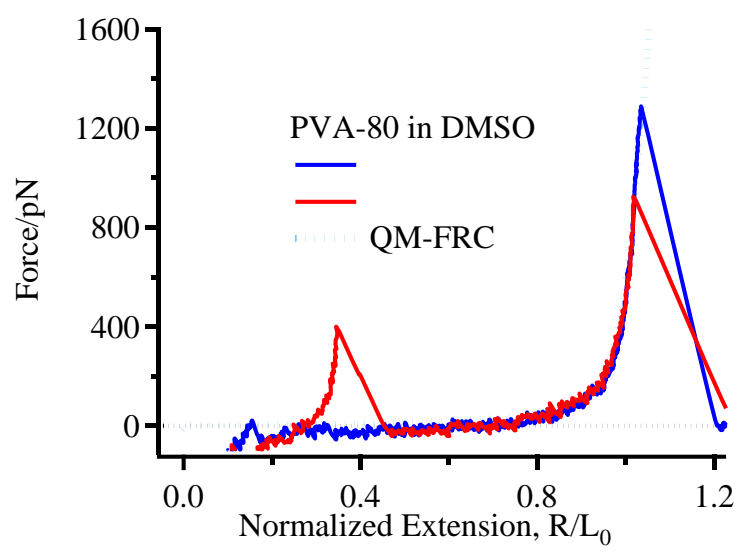

Figure S1. Normalized F-E curves of PVA-80 obtained in DMSO and the QM-FRC fitting curve of PE (blueness dotted line). 


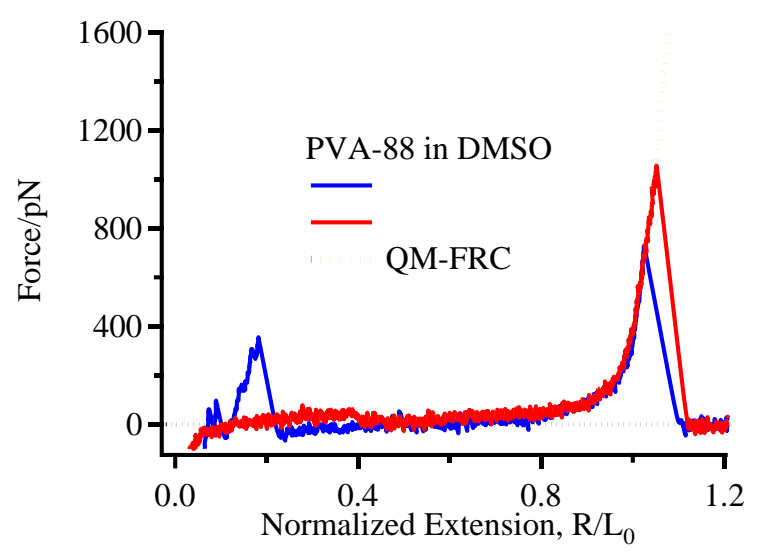

Figure S2. Normalized F-E curves of PVA-88 obtained in DMSO and the QM-FRC fitting curve of PE (orange dotted line).

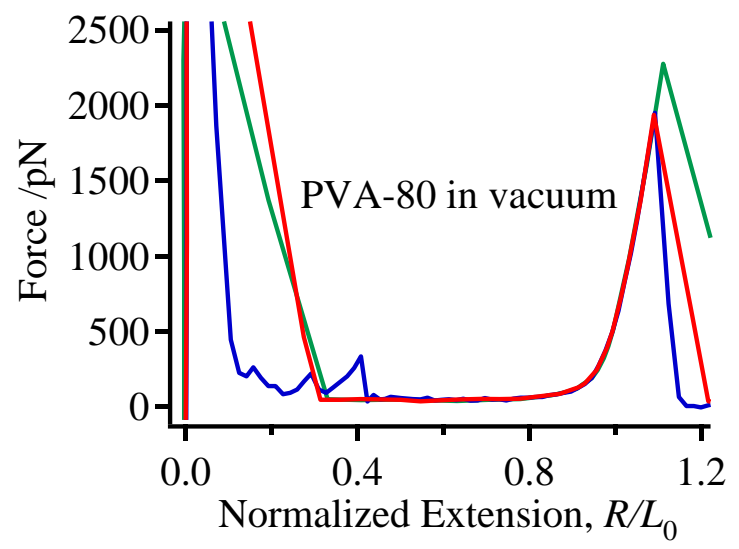

Figure S3. Normalized F-E curves of PVA-80 obtained in high vacuum.

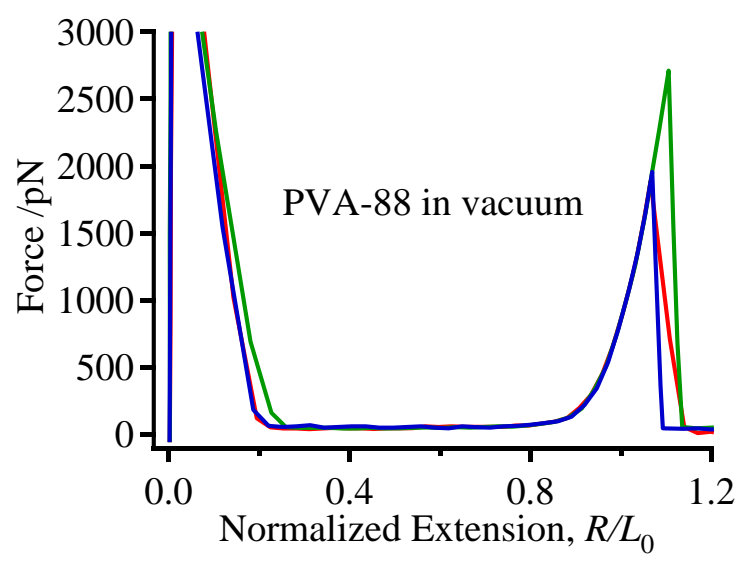

Figure S4. Normalized F-E curves of PVA-88 obtained in high vacuum. 


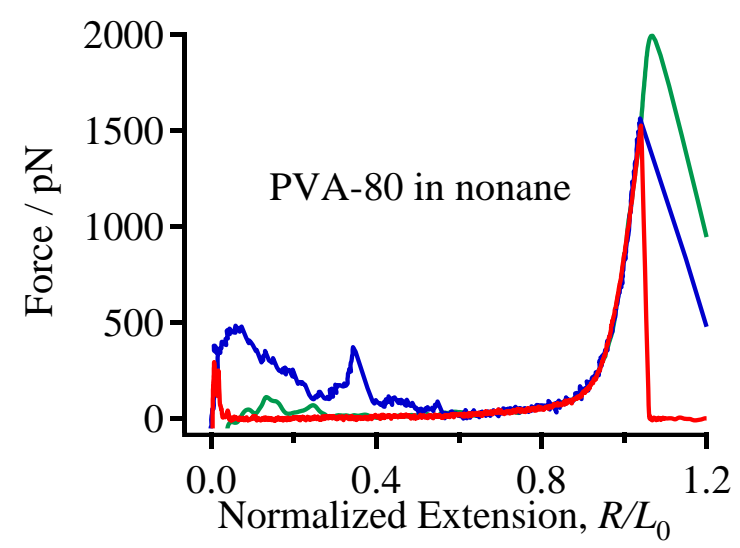

Figure S5. Normalized F-E curves of PVA-80 obtained in nonane.

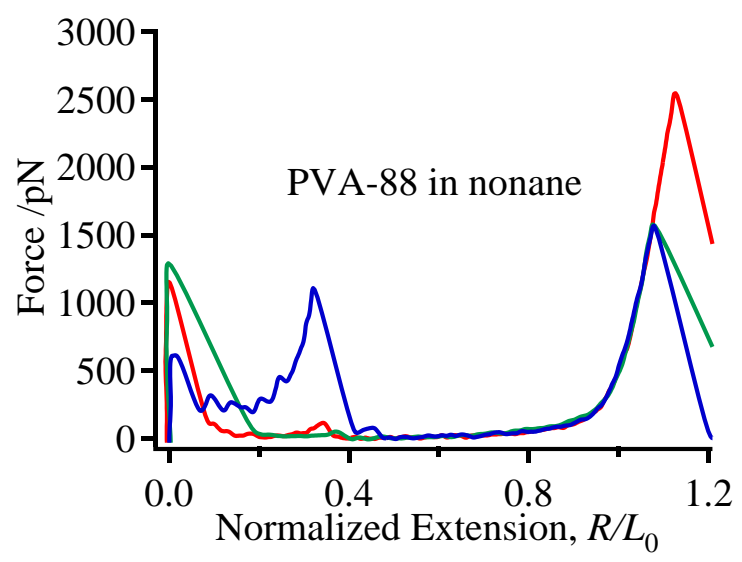

Figure S6. Normalized F-E curves of PVA- 88 obtained in nonane.

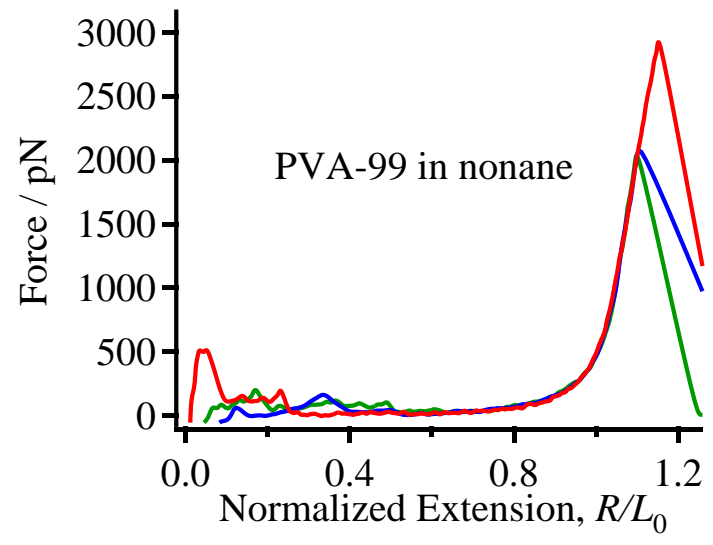

Figure S7. Normalized F-E curves of PVA-99 obtained in nonane. 


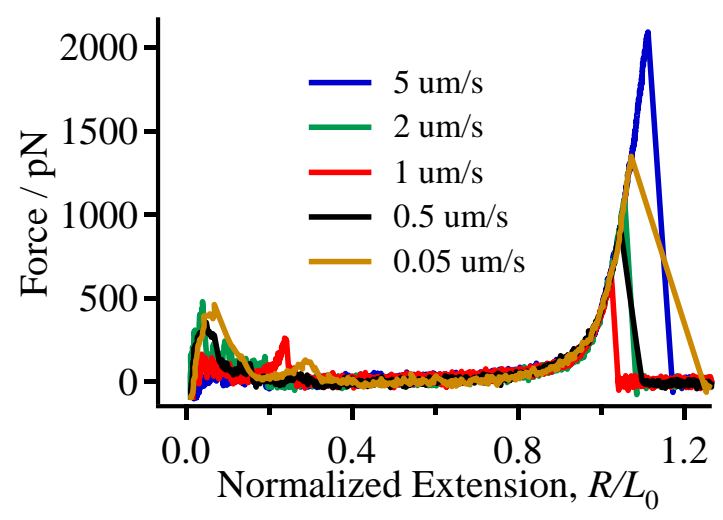

Figure S8. Normalized F-E curves of PVA- 99 obtained in nonane at various pulling speeds $(0.05$, $0.5,1,2$, and $5 \mu \mathrm{m} / \mathrm{s})$.

\section{References}

(1) Wang, K.; Pang, X.; Cui, S., Inherent Stretching Elasticity of a Single Polymer Chain with a Carbon-Carbon Backbone. Langmuir 2013, 29, 4315-4319.

(2) Hugel, T.; Rief, M.; Seitz, M.; Gaub, H. E.; Netz, R. R., Highly Stretched Single Polymers: Atomic-Force-Microscope Experiments versus Ab-Initio Theory. Phys. Rev. Lett. 2005, 94, 048301.

(3) Grandbois, M.; Beyer, M.; Rief, M.; Clausen-Schaumann, H.; Gaub, H. E., How Strong is a Covalent Bond? Science 1999, 283, 1727-1730. 